\title{
FIVE ROOTS PATTERN OF MEDIAN NERVE FORMATION
}

\author{
Konstantinos Natsis, George Paraskevas, Maria Tzika
}

Department of Anatomy, Faculty of Health Sciences, Medical School, Aristotle University of Thessaloniki, Thessaloniki, Greece

Summary: An unusual combination of median nerve's variations has been encountered in a male cadaver during routine educational dissection. In particular, the median nerve was formed by five roots; three roots originated from the lateral cord of the brachial plexus joined individually the median nerve's medial root. The latter (fourth) root was united with the lateral (fifth) root of the median nerve forming the median nerve distally in the upper arm and not the axilla as usually. In addition, the median nerve was situated medial to the brachial artery. We review comprehensively the relevant variants, their embryologic development and their potential clinical applications.

Keywords: Median nerve; Formation; Five roots; Brachial plexus

The median nerve $(\mathrm{MN})$ as it is widely known is formed by the union of the lateral root coming from the lateral cord and the medial root arising from the medial cord of the brachial plexus. These two roots embrace the third portion of the axillary artery (AA) joining each other anterior or lateral to it (21). MN's variability has been well documented in the literature and concerns the number of its roots, the level of its formation and its positioning with respect to the AA. Its normal pattern of formation by two roots has been mentioned varying between $48-88.3 \%(9,13)$, whereas its formation by three or very rarely four roots has been described as well (14, 17). In addition, low MN's distal to the axilla has been found in an incidence of $3.5 \%$ to $40 \%$ of the specimens $(7,14)$, whilst the MN's anterior or medial positioning in regards to AA has been prescribed in the literature (13).

Such variations of the $\mathrm{MN}$ regarding its formation can potentially confuse the surgeon intervening in the axilla and arm region, as well as the anesthetist during axillary block anesthesia. Moreover, lesion of MN's additional roots can alter the expected clinical manifestations. In the current study, we display a very rarely detected in the literature combination of a MN with five roots, placed medially to the AA and brachial artery and formed in the arm region.

\section{Case report}

During routine educational dissection on the left cervical, axilla and arm region of a 78-year-old male cadaver, we came across an unusual combination of variations concerning the ipsilateral MN. After meticulous dissection of the left axilla including removal of the left clavicle, resection of the major as well as the minor pectoral muscles and careful dissection of the connective and adipose tissue of the axillary cavity, we detected the precise pattern of MN's formation, the level of its formation as well as the relationship of $\mathrm{MN}$ to the adjacent AA and brachial artery. It was found that the left MN was formed by five roots instead of two, as is expected normally. Specifically, three roots originated from the lateral cord of the brachial plexus approximately at the level of the coracoid process coursing obliquely anterior to the AA and joining individually the medial root of the MN to form immediately the so-called main medial root of the $\mathrm{MN}$, that constitute the fourth MN's root. The trunk of the $\mathrm{MN}$ was formed by the union of the aforementioned main medial root and the lateral (fourth) root of $\mathrm{MN}$ at the level of the upper third of the arm. The lateral root of MN travelled in front of the AA, directing ultimately medial to the AA. The trunk of MN was situated medial to the brachial artery (Fig. 1). The cause of our specimen's death was unrelated to the current study, whereas no other variants, pathologies or evidence of previous surgical procedures on the relevant regions were present. Moreover, the contralateral brachial plexus and median nerve did not display any variation as regards their formation and topography. Our findings were repeatedly documented by photographs.

\section{Discussion}

The MN has two roots from the lateral and medial cords of the brachial plexus, which embrace the third part of the AA, uniting anterior or lateral to it (21). The medial root crosses in front of the AA. Ultimately, MN trunk is formed at the lower border of the axilla (12). Apart from the abovementioned usual detected MN's pattern of formation, $\mathrm{MN}$ can be formed by the union of three roots, two lateral and one medial $(10,17)$. Such an incidence has been noticed in $11.7 \%(13), 14 \%(8), 26 \%$ (7) or as high as $52 \%(9)$. Occasionally, the third additional lateral root was derived 


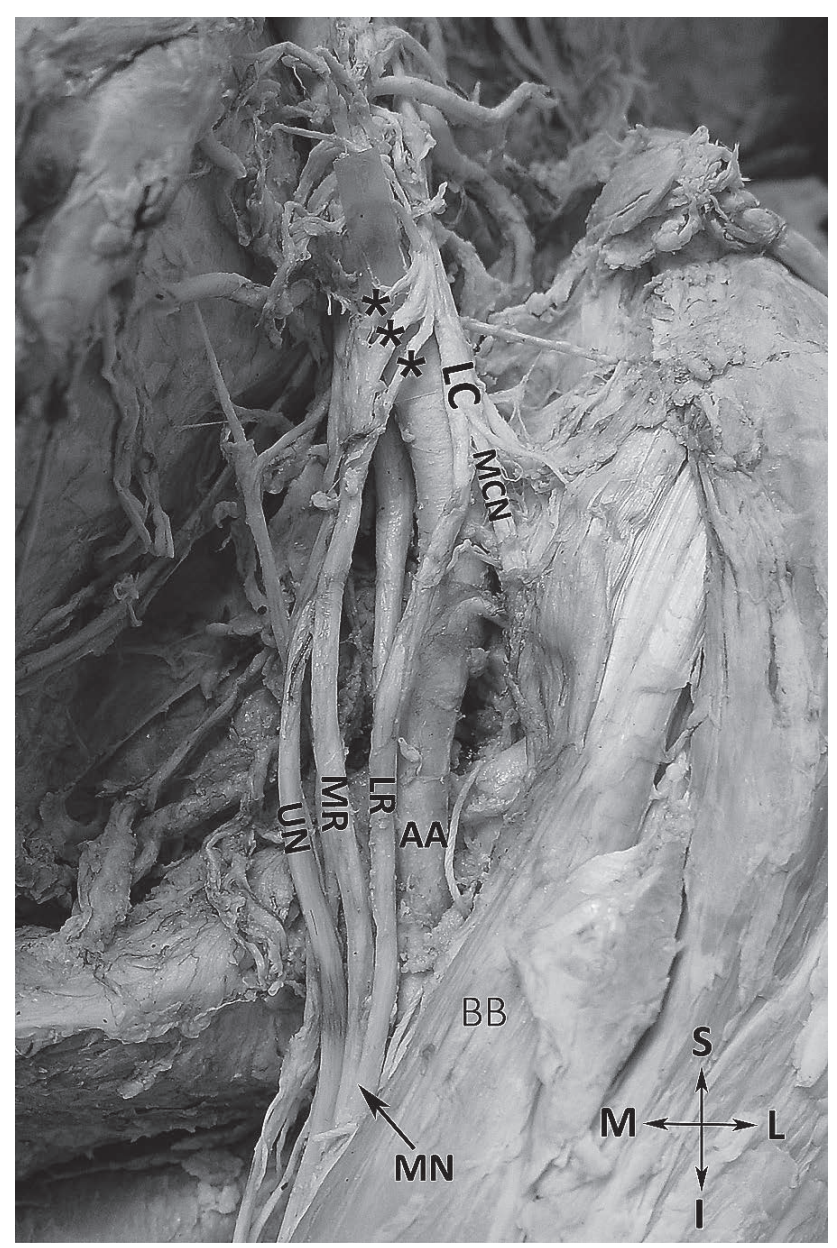

Fig. 1: The left median nerve (MN) is shown forming by the junction of the lateral root (LR) and medial root (MR) of the MN that are derived from the lateral (LC) and medial cord of the brachial plexus, respectively. The medial root receives three additional roots (asterisks). (AA: axillary artery, $\mathrm{MCN}$ : musculocutaneous nerve, $\mathrm{UN}$ : ulnar nerve, BB: biceps brachii muscle, S: superior, I: inferior, M: medial, L: lateral).

from the anterior division of the middle trunk of the brachial plexus $(3.08 \%)$ as noted in a material of infants by Uzun et al. (19). It must be emphasized that many investigators consider as the additional lateral root of $\mathrm{MN}$ the connecting branch from musculocutaneous nerve to MN. The formation of MN from one lateral root and two medial roots from the medial cord of brachial plexus has been mentioned as well (15). Very rarely the MN has been found to be formed by the union of four roots. Budhiraja et al. observed the formation of $\mathrm{MN}$ from four roots in $3.57 \%$ of their material. They noted that the two additional lateral roots took their origin, the one from the lateral cord and the other from the musculocultaneous nerve (6). The two unusual lateral roots in a case report of Uzun et al. were noted to take their origin from the musculocutaneous nerve and joined the MN (20), whereas Pandey et al. (14), Satyanarayana et al. (18) and
Kolagi et al. (11) observed three roots from the lateral cord of the brachial plexus to be passing obliquely anterior to the AA and joining separately with the medial root of the MN. Our case report constitutes to the best of our knowledge a unique, presumably the first described in the literature, case of MN's formation from five roots combined with the distal site of MN's formation in the region of arm and the medial location of $\mathrm{MN}$ in relation to the brachial artery.

In some instances there are additional roots of $\mathrm{MN}$ derived from the lateral cord of the brachial plexus or the MN's lateral root communicating either with the medial cord of the brachial plexus or with the MN's medial root that have been termed "communicating branches" $(10,13$, 14), or "interconnecting branches" (2) or "intercordal neural communications" (4). Badawoud et al. noticed the presence of two such interconnecting branches uniting the two roots of the $\mathrm{MN}$ in $2.1 \%$ and the presence of one interconnecting branch joining the MN's roots in $6.3 \%$ of their specimens (2). Pandey et al. mentioned the existence of one or two communicating branches from the lateral cord to the medial root of $\mathrm{MN}$ in $2.3 \%$ of their cases (14). Interestingly, Goyal et al. observed the presence of a unique communicating branch displaying a recurrent course (10). Apparently, these communicating branches in most cases represent additional roots of the MN. Such communicating branches or additional MN's roots could be prone to lesion during radical neck dissections and surgical procedures undertaken into the area of axilla and the upper arm (19). Such branches could explain unusual sensory loss after injury or surgical intervention in the axilla region $(9,10)$ and their knowledge is crucial during interpretation of neurophysiologic examination of the upper limb. Moreover, these anastomosing branches or additional MN's roots may result in AA's compression reducing the blood supply to the upper limb $(2,3)$.

The site of union between the lateral and the medial root of the MN is quite variable and has been found as far down as the elbow (5). Pandey et al. found low fusion of two roots of the MN in $3.5 \%$ of the cadavers (14), whereas Fazan et al. observed the MN's formation in the region of the arm in a high prevalence of 7\% (9). Similarly, Nasr mentioned that $\mathrm{MN}$ began at the level of the coracobrachialis muscle insertion in $6.7 \%$ of the subjects (13). However, Budhiraja et al. reported that such low origin of $\mathrm{MN}$ is detected in higher incidence, thus $17.3 \%$ (6). Similarly, Channabasanagouda et al. observed the MN's formation in the arm's region with an extremely high incidence of $40 \%$. In particular, such MN's formation was located in the upper third of the arm in $20 \%$, in the middle third in $12 \%$ and in the lower third of the arm in $4 \%$ of the studied specimens (7). The abovementioned authors stated that such ectopic MN's formation might confuse the operative surgeon during surgical intervention in the arm or the anesthetist while performing block anesthesia.

The AA is clasped between the medial and lateral roots of brachial plexus, the medial one crossing in front of the vessel. The commencement of the MN is thus lateral to the artery (12). Otherwise, the two MN's roots embrace the third part 
of AA uniting anterior or lateral to it (21). $\mathrm{MN}$ is formed on the anterior surface of AA in $1.53 \%$ (6) or $8.3 \%$ (13) or $10 \%$ (7). Such anterior relationship of the MN and AA possesses clinical value, since $\mathrm{MN}$ roots potentially compress the AA including ischemic symptomatology. Occasionally, $\mathrm{MN}$ has been noted forming posterior to the AA $(5,13)$. Furthermore, as it occurs in our case, the MN can course across the medial surface of the AA and the initial portion of the brachial artery, a relationship not so infrequently detected, since it can be noted in an incidence of $4.7 \%$ (14) or $6 \%$ (7) or $13.3 \%$ (13).

As regards the embryologic MN's development the ventral root fiber can be seen growing out toward the end of the fourth week, whereas slightly later in the fifth week the ganglia and dorsal root fiber anlages are developed. At the tenth millimeters stage a typical spinal nerve with its ventral and dorsal ramus become evident. The nerves supplying the arm are clearly indicated in embryos of five weeks. The trunks of the last four cervical, together with the first thoracic nerves unite into the primitive brachial plexus (1). The contact between these nerves and the differentiating mesodermal condensations is mandatory for accomplishing the functional differentiation. Any disturbance of coordination of these processes may result in variations in morphology of brachial plexus $(4,16)$.

Conclusively, such a combination of MN's variants should be kept in mind on behalf of the surgeons and the anesthetists dealing with the region of axilla and arm. In particular, the additional roots of MN could induce AA's compression resulting in ischemic disturbances of the ipsilateral upper limb, whereas its accidental laceration could lead in unexpected clinical signs and symptoms. Furthermore, the awareness of the atypical formation and location of $\mathrm{MN}$ is critical for the anesthetist performing brachial plexus block, as well as for the plastic, vascular, orthopedic surgeon, traumatologist and neurosurgeon operating in the region of the axilla.

\section{References}

1. Arey LB. Developmental Anatomy. A textbook and laboratory manual of embryology. 6th edition. Philadelphia: W.B. Saunders Co 1960, p. 502-504.

2. Badawoud MHM. A study on the anatomical variation of the median nerve formation. Bahrain Med Bull 2003; 25(4): 1-5.

3. Bala A, Sinha P, Tamang BK, Sarda RK. Anatomical variation: median nerve formation - a case vignette. J Clin Diag Res 2014; 8(6): AD03-AD04.

4. Baliyan R, Mehta V, Arora J, Nayyar AK, Suri RK, Rath G. Unilateral intercordal neural communication coexistent with variant branching pattern of posterior cord of brachial plexus. Acta Medica (Hradec Králové) 2011; 54(3): 131-134.

5. Bergman RA, Thompson SA, Afifi AK, Saadeh FA. Compendium of human anatomic variation. Text, atlas and world literature. Munich: Urban und Schwarzenberg 1988, p. 141 .

6. Budhiraja V, Rastogi R, Asthana AK, Sinha P, Krishna A, Trivedi V. Concurrent variations of median and musculocultaneous nerves and their clinical correlation a cadaveric study. Ital J Anat Embryol 2011; 166: 67-72.

7. Channabasanagouda, Shrish P, Shinde V, Jevoor PS, Nidoni M. A study of anatomical variations of median nerve in human cadavers. IJBR 2013; 4(12): 682-690.

8. Egleseder E, Goldman M. Anatomical variations of the musculocutaneous nerve in the arm. Am J Orthop 1997; 26: 777-780.

9. Fazan VPS, Amadeu AdS, Caleffi AL, Filho OAR. Brachial plexus variations in its formation and main branches. Acta Cir Bras 2003; 18(5): 14-18.

10. Goyal N, Harjeet, Gupta M. Bilateral variant contributions in the formation of median nerve. Surg Radiol Anat 2005; 27: 562-565.

11. Kolagi SI, Rairam GB, Herur A. Multiple variations of median nerve with persistent median artery. A case report and review of literature. Anatomica Karnataka 2010; 4(1): 44-46.

12. McMinn R. Last's Anatomy. Regional and Applied. 8th edition. Edindurgh : Churchill Livingstone 1990, p. 82

13. Nasr AY. Morphology and clinical significance of the distribution of the median nerve within the arm of human cadavers. Neurosciences 2012; 17(4): 336-344.

14. Pandey SK, Shukla VK. Anatomical variations of the cords of brachial plexus and the median nerve. Clin Anat 2007; 20: 150-156.

15. Paranjape V, Swamy PV, Mudey A. Variant median and absent musculocultaneous nerve. J Life Sci 2012; 4(2): 141-144.

16. Saddler TW. Muscular system. In: Langman's Medical embryology. 10th edition. Philadelphia: Lippincott Williams and Wilkins 2006, p. 146-147.

17. Sargon MF, Uslu SS, Celik HH, Aksit D. A variation of the median nerve at the level of the brachial plexus. Bull Assoc Anat 1995; 79: 25-26.

18. Satyanarayana N, Vishwakarma N, Kumar GP, Guha R, Datta AK, Sunitha P. Rare variations in the formation of median nerve - embryological basis and clinical significance. Nepal Med Coll J 2009; 11(4): 287-290.

19. Uzun A, Bilgic S. Some variations in the formation of the brachial plexus in infants. Tr J Med Sci 1999; 29: 573-577.

20. Uzun A, Seelig LLJr. A variation in the formation of the median nerve: communicating branch between the musculocutaneous and median nerves in man. Folia Morphol (Warsz) 2011; 60: 99-101.

21. Williams PL (ed.). Gray's Anatomy. The anatomical basis of Medicine and Surgery. 38th edition. Edinburgh: Churchill Livingstone 1995, p. 1270.

Received: 02/12/2015

Accepted in revised form: 28/01/2016

\section{Corresponding author:}

Dr. G. Paraskevas, Assoc. Prof. of Anatomy, Department of Anatomy, Faculty of Health Sciences, Medical School, Aristotle University of Thessaloniki, Thessaloniki, Greece; email: g_paraskevas@yahoo.gr 